\title{
INFLUÊNCIA DA APLICAÇÃO DE VÁCUO NOS PROCESSOS DE SALGA DE CARNE BOVINA
}

\author{
M. BAMPI ${ }^{1}$, F. C. SCHMIDT ${ }^{2}$, J.B. LAURINDO ${ }^{1}$
}

\begin{abstract}
${ }^{1}$ Departamento de Engenharia Química e Engenharia de Alimentos. Centro Tecnológico. Universidade Federal de Santa Catarina. EQA/CTC/UFSC, Florianópolis - SC, Brasil.

${ }^{2}$ Departamento de Engenharia de Alimentos. Instituto de Ciências Tecnológicas e Exatas. Universidade Federal do TriânguloMineiro. DEA/ICTE/UFTM, Uberaba - MG, Brasil.

E-mail para contato: eng_marlene@ hotmail.com
\end{abstract}

\begin{abstract}
RESUMO - O objetivo deste estudo foi avaliar a influência da aplicação de pulsos de vácuo (PV) no processo de salga úmida (SU) e salga mista (SM) de cortes de carne bovina. Os processos SU (imersão em solução saturada de $\mathrm{NaCl}$ ) e SM (20 minutos de SU seguido por salga seca) realizados à pressão atmosférica (Patm) foram comparados aos processos de salga com PV de 50 $\mathrm{mmHg}$ nos primeiros 20 minutos da etapa SU. Todos os processos foram realizados a temperatura $10{ }^{\circ} \mathrm{C}$ por 6 horas. A influência da aplicação de PV foi avaliada através dos parâmetros: perda de massa total $(W R)$, perda de água $(W L)$, ganho de sal $(S t G)$ e atividade de água $\left(\mathrm{a}_{\mathrm{w}}\right)$. No processo de $\mathrm{SU}$, a aplicação de $1 \mathrm{PV}$ aumentou em $23,9 \%$ a $W L, 3,8 \%$ o $S t G, 11,5 \%$ a $W R$ e reduziu em $1,1 \%$ a $\mathrm{a}_{\mathrm{w}}$. A aplicação de 3PV ocasionou um aumento de $13,3 \%$ na $W L, 5,8 \%$ no $S t G$, $1,3 \%$ na $W R$ e reduziu em $1,5 \%$ a $\mathrm{a}_{\mathrm{w}}$ em relação a $\mathrm{SU}$ à Patm. Por outro lado, a aplicação de 1PV na SM reduziu em 23,5\% a $W L, 23,8 \%$ a $W R$ e aumentou em $0,6 \%$ o $S t G$ e $0,5 \%$ a a $\mathrm{a}_{\mathrm{w}}$, quando comparado aos processos de SM à Patm. Dessa forma, a aplicação de PV durante a SU é uma alternativa para incrementar a desidratação e reduzir o tempo de salga de cortes de carne bovina.
\end{abstract}

\section{INTRODUÇÃO}

O processo de salga é um dos métodos de conservação mais antigo e amplamente utilizado no processamento de produtos cárneos. A influência da salga na conservação do alimento se deve basicamente à capacidade do sal em reduzir os valores de atividade de água $\left(\mathrm{a}_{\mathrm{w}}\right)$ do produto (Barat et al., 2011; Albarracín et al., 2011). Os métodos mais comuns de salga de produtos cárneos, tanto na produção artesanal como na industrial, são a salga seca (SS), a salga úmida (SU) e a salga mista (SM) (Gava et al., 2008). Nos últimos anos, a aplicação de vácuo nos processos de salga tem sido reportada como alternativa para reduzir o tempo de salga, além de promover uma distribuição mais homogênea de sal no produto (Chiralt et al., 2001; Hofmeister et al., 2005; Schmidt et al., 2008).

O processo de impregnação a vácuo (IV) de alimentos porosos consiste na troca do gás e do líquido nativo presente no interior dos poros por um líquido externo. Isso se faz pela ação de gradientes macroscópicos de pressão promovidos pela aplicação de vácuo, seguida pelo restabelecimento da pressão atmosférica em uma câmara contendo o produto imerso em uma solução (Chiraltet al., 2001). No primeiro passo do processo de IV, submete-se o 
sistema a uma pressão subatmosférica $\left(\mathrm{P}_{1}\right)$ por um dado período de tempo $\left(\mathrm{t}_{1}\right)$, onde o gás ocluso nos poros se expande, até o equilíbrio com a pressão imposta ao sistema, saindo do produto e drenando parte do líquido nativo presente no interior dos poros. Uma vez alcançado o equilíbrio das pressões do sistema, uma quantidade de líquido penetra nos poros devido à ação das forças capilares. Após este período, a pressão atmosférica $\left(\mathrm{P}_{2}\right)$ é restabelecida e o produto é mantido imerso na solução por um tempo $t_{2}$. Nesta segunda etapa, o gás residual presente no interior da amostra é comprimido, conduzindo a impregnação dos poros do produto pela solução externa (Chiralt et al., 2001). Esse fenômeno implica uma mudança composicional rápida no produto, com modificação das condições para a transferência de massa. Fito (1994) propôs o primeiro modelo matemático destinado à predição da impregnação a vácuo, denominado pelo autor de Mecanismo Hidrodinâmico (HDM).

Diante do exposto, o objetivo deste trabalho foiavaliar a influência da aplicação de pulsos de vácuo (PV) no processo de salga úmida (SU) e salga mista (SM) de cortes de carne bovina.

\subsection{MATERIAL E MÉTODOS}

Matéria-prima, preparo das amostras e soluções salinas: Os cortes de carne bovina in natura (acém) utilizados nos experimentos foram adquiridos no comércio local de Florianópolis (SC) e cortados em formato de paralelepípedos com dimensões de 8,0 x 8,0 x $1,5 \mathrm{~cm}$ (comprimento $\mathrm{x}$ largura $\mathrm{x}$ espessura) com massa de aproximadamente $150 \mathrm{~g}$. As soluções salinas foram preparadas com cloreto de sódio ( $\mathrm{NaCl}, \mathrm{P} . \mathrm{A}$.) e água destilada. Uma razão mássica de 1:10 de amostras:salmoura foi utilizada na etapa de salga úmida, para evitar mudanças significativas na concentração de sal durante os ensaios

Sistema experimental utilizado na SU e SM: A salga úmida foi realizada em um dispositivo experimental composto por uma câmara de aço inoxidável encamisada (volume interno de 53,5 L), conectada a uma bomba de vácuo com vazão nominal de $350 \mathrm{~m}^{3} \mathrm{~h}^{-1}$ (DVP, modelo LC. 305, Itália) e a uma bomba de agitação de líquidos com vazão de $5.000 \mathrm{~L}$ $\mathrm{h}^{-1}$ (Bombinox, modelo BL 05, Brasil). O monitoramento da pressão na câmara foi realizado através de um transmissor de pressão (Wärme do Brasil, modelo WTP-4010) conectado a um microcomputador. A temperatura no interior da câmara foi controlada através da circulação de água resfriada na camisa, proveniente de um banho termostático (Quimis, Modelo Q214M2). A SS foi realizada em um recipiente plástico (PVC) com uma grade suspensa na altura de $5 \mathrm{~cm}$ da base,de modo que as amostras não ficassem em contato com a água exsudada durante o processo.

Processos de salga à Patm e com PV: Para o estudo da transferência de massa durante a salga das amostras de carne à pressão atmosférica (Patm) foram avaliados os seguintes processos: (i) SU e (ii) SM. No processo de SU, as amostras foram submersas em solução saturada de $\mathrm{NaCl}(26 \% \mathrm{~m} / \mathrm{m})$ por seis horas. No processo de $\mathrm{SM}$ as amostras foram submetidas à SU nos primeiros 20 minutos de processo. Após esse período, as mesmas foram retiradas da salmoura, envoltas por uma camada de sal de aproximadamente $5 \mathrm{~mm}$ e depositada sobre uma grade suspensa em um recipiente plásticopor 5 horas e 40 minutos, totalizando 6 horas de salga.

Nos processos de salga com aplicação de PV, a influência do vácuo na cinética de transferência de massa nos processos de salga (SU e SM) foi avaliada em duas etapas. Na primeira etapa foi aplicado um pulso de vácuo (1PV), tanto no processo de SU quanto no processo de SM. Esse correspondeu à aplicação de uma pressão de $50 \mathrm{mmHg}$ nos primeiros 
20 minutos de salga, seguido do restabelecimento da Patm por cinco minutos. Na segunda etapa foram aplicados três pulsos de vácuo (3PV), somente no processo de SU. Os 3PV são caracterizados por uma sequência de alterações de pressão de $50 \mathrm{mmHg}$ por cinco minutos, seguidos do restabelecimento da Patm por dois minutos.

Todos os processos foram realizadosa temperatura $10{ }^{\circ} \mathrm{C}$ por 6 horas eem duplicata, sendo utilizado um número de amostras suficiente para que em cada tempo de amostragem fossem retiradas três amostras(as quais foram analisadas individualmente).Na primeira hora de salga foram retiradas amostras a cada 20 minutos e posteriormente a cada hora, até o final do experimento.

Análises físico-químicas: $\mathrm{O} \mathrm{pH}$ das amostras foi determinado utilizando potenciômetro digital com eletrodo para sólidos (marca Analion, modelo PH- 730, Brasil). A variação de cor foi avaliada através de um colorímetro (Hunterlab, Miniscan EZ), com observador de $10^{\circ}$ e iluminante D65, utilizando o sistema de leitura CIE (Commission Internationale d'Eclairage) L*, a*, b*, assumindo como referência a cor da carne in natura. A umidade ( $X_{a}$ ) das amostras in natura edassubmetidas aos diferentes processos de salga foi determinada de acordo com o método gravimétrico da AOAC (2007) (ISO R-1442). A atividade de água $\left(\mathrm{a}_{\mathrm{w}}\right)$ foi determinada através de um higrômetro (Decagon Devices inc., Pullman, Aqualab Model Series 3). A concentração de $\mathrm{NaCl}$ foi calculada a partir da quantificação de cloretos, determinado em analisador automático de cloretos (Cole Parmer, modelo 926) de acordo com a metodologia apresentada por Aliño et al. (2011).

Parâmetros de processo: A perda de água $(W L)$, perda de massa total $(W R)$ e o ganho de sal ( $S t G$ ) pelas amostras no decorrer de 6 horas de salga foram determinados através das Equações 1, 2 e 3, respectivamente.

$$
\begin{aligned}
& W L=\frac{\left(M X_{a}\right)-\left(M^{0} X_{a}{ }^{0}\right)}{M^{0}} \times 100 \\
& W R=\frac{M-M^{0}}{M^{0}} \times 100 \\
& S t G=\frac{\left(M X_{\mathrm{NaCl}}\right)-\left(M^{0} X_{\mathrm{NaCl}}{ }^{0}\right)}{M^{0}} \times 100
\end{aligned}
$$

em que $M$ é a massa após um tempo $t$ de processo, $M^{\circ}$ é a massa da amostra no tempo inicial $(\mathrm{t}=0), X_{a}$ e $X_{\mathrm{NaCl}}$ representam o teor de água e o teor de sal, respectivamente, após um tempo $t$ de processo e $X_{a}{ }^{0}$ e $X_{\mathrm{NaCl}}{ }^{0}$, os teores iniciais de água e sal na amostra $(\mathrm{em} \mathrm{t}=0)$. Para verificar se há diferença significativa entre os parâmetros determinados nos diferentes processos estudados foi realizada uma análise de variância simples (ANOVA one way). Nos casos em que há diferença significativa, foi aplicado o teste de Tukey com um nível de significância de 5\%. O software utilizado nas análises estatísticas foi o Statistica® 7.0.

\section{RESULTADOS E DISCUSSÕES}

Os valores médios das propriedades físico-químicas da carne bovina in natura são apresentados na Tabela 1 . 
Tabela 1 Caracterização físico-química das amostras de carne bovina in natura.

\begin{tabular}{lc}
\hline Propriedades & Valores \pm Desvio padrão \\
\hline Umidade (g água/g amostra) & $0,75 \pm 0,01$ \\
$\mathrm{a}_{\mathrm{w}}$ & $0,99 \pm 0,00$ \\
$\mathrm{pH}$ & $5,42 \pm 0,14$ \\
Teor de $\mathrm{NaCl}(\mathrm{g}$ de NaCl/g amostra) & $0,16 \pm 0,01$ \\
\hline
\end{tabular}

Os valores obtidos para umidade, $\mathrm{a}_{\mathrm{w}} \mathrm{e} \mathrm{pH}$ foram semelhantes aos encontrados por Alves et al. (2010) em amostras de carne bovina in natura, sendo esses de $0,74 \mathrm{~g}$ água/g amostra para a umidade, 0,99 para a $\mathrm{a}_{\mathrm{w}}$ e 5,65 para o $\mathrm{pH}$. O teor de cloreto de sódio obtido foi semelhante aos valores encontrados na tabela Brasileira de composição de alimentos (Taco 2011), o qual é de aproximadamente $0,13 \mathrm{~g}$ de $\mathrm{NaCl} / \mathrm{g}$ amostra.

\subsection{Influência da aplicação de pulsos de vácuo na transferência de massa durante a salga de cortes de carne bovina.}

As cinéticas de $W L, S t G, W R$ e a variação da $a_{\mathrm{w}}$ das amostras submetidas aos processos de SM e SU à Patm são apresentadas nas Figuras 1, 2, 3 e 4, juntamente com as cinéticas dos processos com aplicação de 1PV e 3PV.

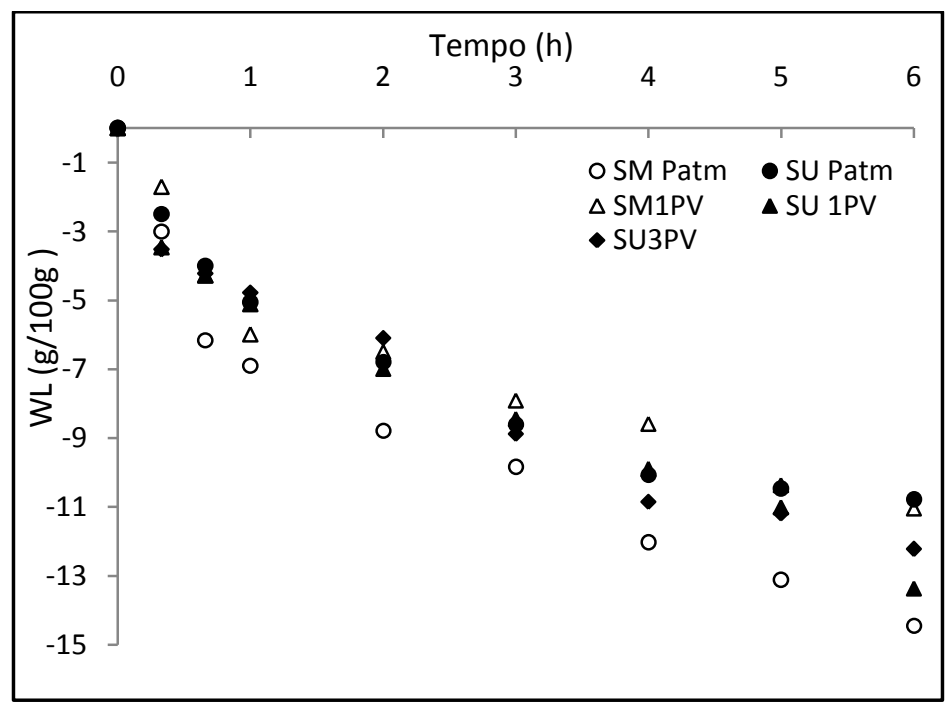

Figura 1- Cinética da $W L$ pelos cortes de carne bovina submetidos aos processos de salga à Patm e com 1PV e 3PV.

Nota-se na Figura1 que, dentre os processos de salga estudados, a SM resultou em maior $W L$ pelas amostras ao final de 6 horas. Isso se deve ao contato direto do $\mathrm{NaCl}$ com as amostras que, em altas concentrações, causa uma intensa desnaturação das proteínas miofibrilares afetando sua capacidade de retenção de água. Com relação aos processos a vácuo, observou-se que aplicação de 1PV no processo de SM ocasionou uma redução de $23,5 \%$ na $W L$ em relação às amostras submetidas ao processo de SM à Patm. Isso pode ser atribuído à impregnação da solução salina devido aos gradientes de pressão provocados pela aplicação de vácuo seguido do restabelecimento da pressão atmosférica. Por outro lado, no 
processo SU a aplicação de 1PV aumentou a WL pelas amostras em 23,9\% em comparação ao processo de SU à Patm. A aplicação de 1PV ocasiona a entrada de solução salina nos espaços entre as fibras e entre os feixes de fibras, aumentando a área de contato para a transferência de água o que, consequentemente, provoca uma maior perda de água ao longo do processo em função da alta concentração de sal na solução. Em relação ao processo de SU com a aplicação de 3PV, observou-se um aumento de $13,3 \%$ na $W L$ em comparação ao processo de SU à Patm. No entanto, a menor perda de água verificada entre o processos de SU com 1 e 3 PV (Figura 1) pode estar associada à influência dos repetidos ciclos de alteração da pressão que modificam a estrutura das amostras.

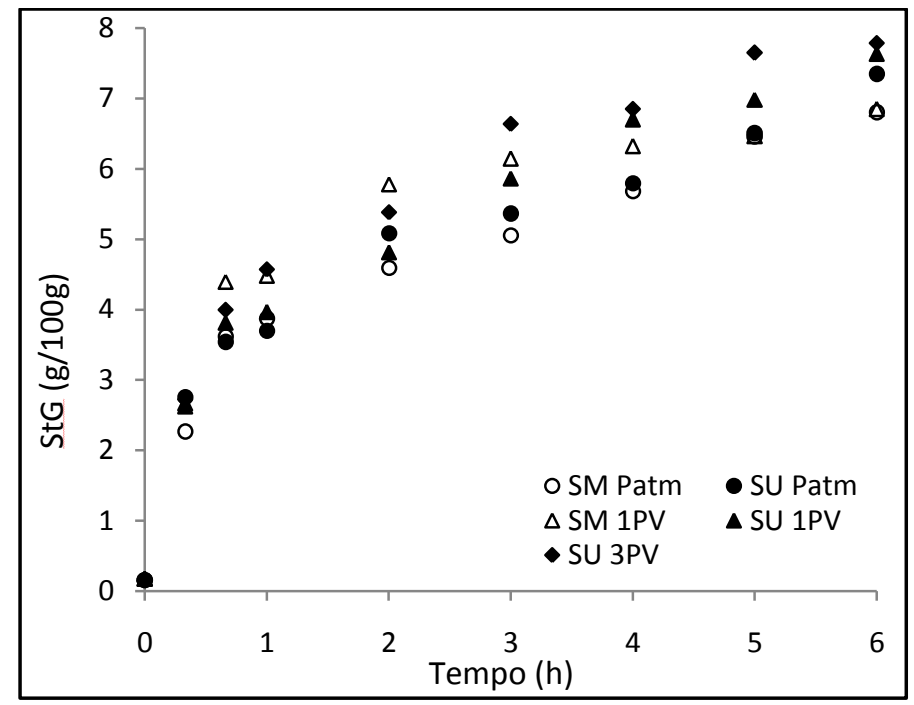

Figura Erro! Nenhum texto com o estilo especificado foi encontrado no documento.. Cinética do $S t G$ pelos cortes de carne bovina submetidos aos processos de salga à Patm e com 1PV e 3PV.

Na Figura 2 observa-se que, após a terceira hora de salga, as amostras submetidas ao processo de SU com 3PV apresentaramos maiores valores de $S t G$. A aplicação de 1PV nos processos de SM resultou em um aumento de apenas 0,6\% no $S t G$ das amostras em relação ao ganho observado para as amostras submetidas ao processo de SM à Patm. Por outro lado, os valores de $S t G$ pelas amostras submetidas ao processo de SU com $1 \mathrm{PV}$ e com 3PV foram de $3,8 \%$ e 5,8\%, respectivamente, maiores que os observadospara o processo de SU à Patm. Destaca-se que no processo de SU as amostras permanecem em contato com a solução salina durante todo processo, enquanto que na SM esse contato foi de apenas 20 minutos. Após esse período, as amostras foram mantidas em contado direto com o sal e o mecanismo difusivo controla o processo de transferência de massa. Schmidt et al. (2008) também observaram um aumento no $S t G$ quando aplicado $\mathrm{PV}$ nos processos de salga úmida de cortes de carne de frango.

Para atingir uma concentração de $\mathrm{NaCl}$ de 3\% nas amostras, através dos processos de SU à Patm, com 1PV e com 3PV são necessários aproximadamente 33, 31 e 28 minutos, respectivamente. Esses resultados representam uma redução de $15 \%$ no tempo de salga das amostras com 3PV em comparação com o processo de SU à Patm. 


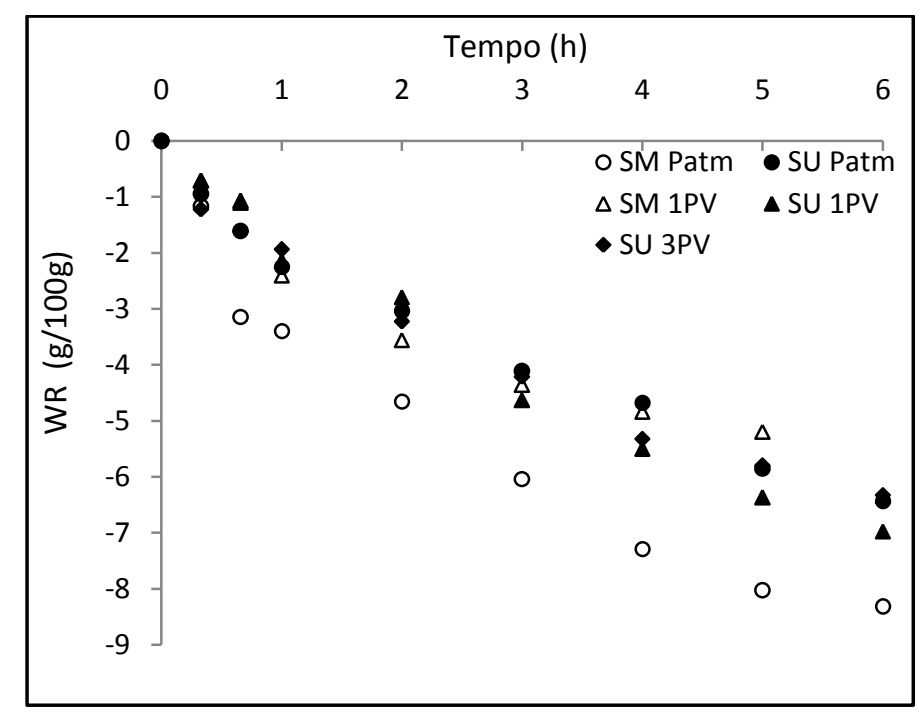

Figura 3 Cinética da $W R$ pelos cortes de carne bovina submetidos aos processos de salga à Patm e com 1PV e 3PV.

Na Figura 3 observa-se que, ao final do experimento de salga, a aplicação de 1PV nos processos de SM ocasionou uma diminuição na $W R$ de aproximadamente $23,8 \%$ em relação ao processo de SM à Patm. No entanto, um comportamento contrário foi observado quando aplicado 1PV e 3PV no processo de SU. Esses ocasionaram um aumento na $W R$ de aproximadamente $11,5 \%$ e 1,3\% respectivamente, em comparação ao processo de SU à Patm. Os resultados de $W R$ corroboram com dados anteriores de $W L$ e $S t G$ (Figuras 1 e 2), onde observou-seque a aplicação de vácuo aumentou a $W L$ e o $S t G$.

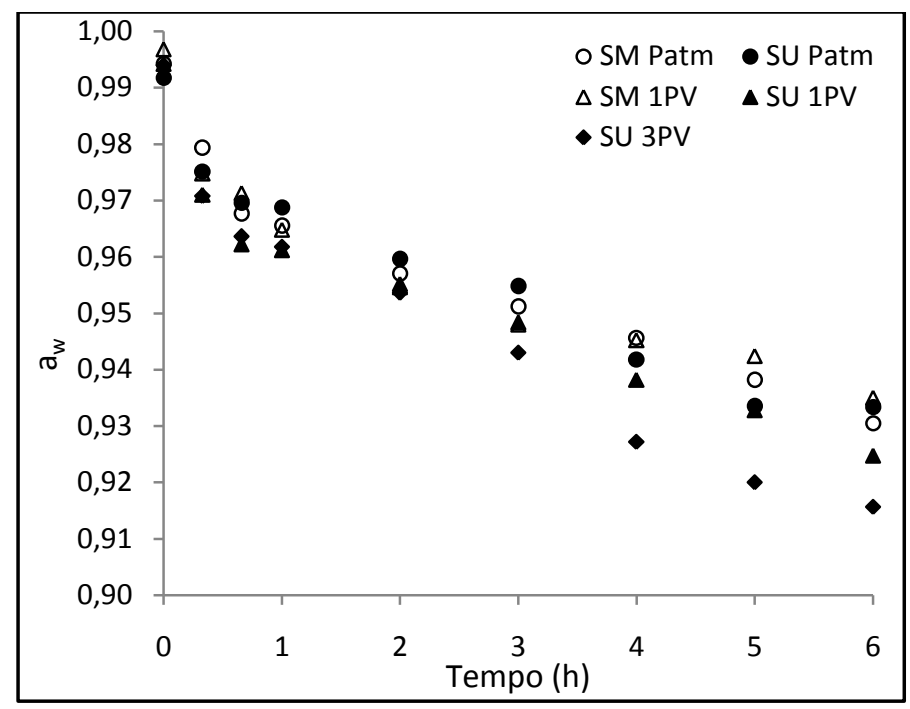

Figura 4 Cinética da variação da $a_{w}$ nos cortes de carne bovina submetidos aos processos de salga àPatm, com aplicação de 1PV e 3PV.

Observa-se na Figura 4 que, após seis horas de salga, as amostras submetidas ao processo de SM com a aplicação de 1PV apresentaram valores de $a_{w}$ menores que os observados para as amostras submetidas ao processo de SM à Patm. Por outro lado, nos processos de SU, a aplicação de $1 \mathrm{PV}$ e $3 \mathrm{PV}$ resultou em uma redução nos valores de $\mathrm{a}_{\mathrm{w}}$ 
quando comparados aos valores das amostras submetidas ao processo à Patm. Isso pode ser explicado pela maior concentração de sal nas amostras submetidas aos processos de SU com aplicação de vácuo.

Na Tabela 2 são apresentados os valores médios de pH e os parâmetros de cor da carne in natura e das amostras submetidas aos processos de SM e SU com aplicação de 1PV e 3PV, ao final de seis horas de salga.

Tabela Erro! Nenhum texto com o estilo especificado foi encontrado no documento..

Resultados das análises de $\mathrm{pH}$ e parâmetros de cor dos cortes de carne bovina in natura e das amostras submetidas aos processos de SM com aplicação de 1PV e SU com 1PV e 3PV.

\begin{tabular}{|c|c|c|c|c|c|}
\hline & & \multirow[t]{2}{*}{ Carne in natura } & $\begin{array}{l}\text { Salga mista } \\
\text { (6 horas) }\end{array}$ & \multicolumn{2}{|c|}{$\begin{array}{l}\text { Salga úmida } \\
\text { (6 horas) }\end{array}$} \\
\hline & & & $1 \mathrm{PV}$ & $1 \mathrm{PV}$ & $3 \mathrm{PV}$ \\
\hline $\mathrm{pH}$ & & $5,20 \pm 0,12^{\mathrm{a}}$ & $5,23 \pm 0,14^{\mathrm{a}}$ & $5,44 \pm 0,13^{\mathrm{a}}$ & $5,41 \pm 0,06^{\mathrm{a}}$ \\
\hline \multicolumn{6}{|c|}{ Cor } \\
\hline & $\mathrm{L}^{*}$ & $42,35 \pm 2,97^{\mathrm{b}}$ & $39,90 \pm 1,68^{\mathrm{ab}}$ & $39,11 \pm 1,51^{\mathrm{a}}$ & $41,68 \pm 1,39^{b}$ \\
\hline & $a^{*}$ & $14,89 \pm 2,59^{c}$ & $10,36 \pm 0,67^{\mathrm{b}}$ & $6,05 \pm 0,62^{\mathrm{a}}$ & $6,92 \pm 0,75^{\mathrm{a}}$ \\
\hline & $\mathrm{b}^{*}$ & $12,75 \pm 2,79^{\mathrm{c}}$ & $7,49 \pm 0,98^{\mathrm{b}}$ & $4,89 \pm 0,98^{\mathrm{a}}$ & $8,54 \pm 1,13^{\mathrm{b}}$ \\
\hline & $\Delta E^{*}$ & & $8,09 \pm 2,18^{\mathrm{a}}$ & $13,46 \pm 3,08^{b}$ & $14,26 \pm 2,23^{b}$ \\
\hline
\end{tabular}

Nota: Valores seguidos de mesma letra, na mesma linha, não diferem entre si pelo teste Tukey com um nível de significância de 5\%.

Para todos os processos analisados, observou-se que os valores dos parâmetros de cor $a^{*}$ (tendência a cor vermelha) e $b^{*}$ (tendência a cor amarela) sofreram uma redução, em relação aos valores observados para amostras in natura. A aplicação de 3PV no processo de SU ocasionou a maior variação de cor das amostras em comparação com os demais processos de salga. Essa maior variação de cor pode ser atribuída a maior solubilização da mioglobina, devido a uma maior impregnação da solução salina nos processos de SU.

\section{CONCLUSÕES}

Os resultados de $S t G$ pelos cortes de carne bovina indicam que a aplicação de $1 \mathrm{PV}$ ou 3PV pode ser utilizada para reduzir os tempos de salga, tanto no processo de SM quanto na SU. A aplicação de $3 \mathrm{PV}$ nos processos de SU resulta em maiores valores de $S t G$ pelas amostras e consequentemente menores valores de $\mathrm{a}_{\mathrm{w}}$, em relação aos demais processos de salga estudados. A maior variação da cor em relação às amostras in natura ocorre quando as amostras são submetidas aos processos de SU.

Os resultados de $W L$ indicam que a aplicação de $1 \mathrm{PV}$ é uma alternativa que pode ser aplicada no processo de SU, quando se busca uma maior perda de água (desidratação), podendo também ser aplicado no processo de SM de cortes de carne bovinos quando se visa menorWL.

\section{REFERÊNCIAS}

ALBARRACÍN, W.; SÁNCHEZ, I.C.;GRAU, R.; BARAT, J.M. Salt in food processing; 
usage and reduction: a review. Int J Food Sci Tech, v.46,p. 1329-1336, 2011.

ALIÑO, M.; FUENTES, A.; FERNÁNDEZ-SEGOVIA, I.; BARAT, J.M. Development of a low-sodium ready-to-eat desalted cod.JFoodEng, v.107, p. 304-310, 2011.

ALVES, L. L.; DELBEM, Á.C.B.; ABREU, U.G.P.; LARA, J.A.F. Avaliação físicoquímica e microbiológica da carne soleada do Pantanal. Ciênc. Tecnol. Aliment, v.30, p. 729-734, 2010.

BARAT, J. M.; BAIGTS, D.; ALINO, M.; FERNANDEZ, F.J.; PEREZ-GARCIA, V.M. Kinetics studies during $\mathrm{NaCl}$ and $\mathrm{KCl}$ pork meat brining.J Food Eng, v.106, p. 102-110, 2011.

BARAT, J.M.; RODRIGUEZ-BARONA, S.; ANDRÉS, A.; FITO, P. Cod salting manufacturing analysis. Food Research International, v. 36, p.447-453, 2003.

CHIRALT, A. et al. Use of vacuum impregnation in food salting process. J Food Eng, v. 49, p. 141-151, 2001.

FITO, P.; CHIRALT, A.; BARAT, J.M.; ANDRÉS,A.; MARTÍNEZ-MONZÓ, J.; MARTÍNEZ-NAVARRETE,N. Vacuum impregnation for development of new dehydrated products. J FoodEng, v. 49, p. 297-302, 2001.

GAVA, A.J.; SILVA, C.A.B. E FRIAS. Tecnologia de alimentos. Princípios e Aplicações. São Paulo: Nobel, 2008. 513p.

HOFMEISTER, L.C.; SOUZA, J.A.R.; LAURINDO, J.B. Use of dyed solutions to visualize different aspects of vacuum impregnation of Minas cheese. Food SciTechnol-Leb, v. 38, n. 4, p. 379-386, 2005 b.

SCHMIDT, F.C.; CARCIOFI, B.A.M.; LAURINDO, J.B. Salting operational diagrams for chicken breast cuts: Hydration-dehydration. J FoodEng, v.88, p. 36-44, 2008.

NEPA. (Núcleo de Estudos e Pesquisas em Alimentação). Tabela brasileira de composição de alimentos - TACO. 4.ed. 2011. Online. Disponível em: <http://www.unicamp.br/nepa/taco/contar/taco_4_edicao_ampliada_e_revisada.pdf?arquivo =taco_4_versao_ampliada_e_revisada.pdf > 